\title{
An Approach to Design and Reduce Cost of IP Telephony using SIP TRUNKING
}

\author{
Dhruv Saxena \\ M.Tech Scholar \\ Dept. of Electronics \& \\ Communication, NIIST, Bhopal
}

\author{
Puran Gour \\ Head \& Professor \\ Dept. of Electronics \\ \&Communication, NIIST, Bhopal
}

\author{
Brahmi Shrman \\ Assistant Professor \\ Dept. of Electronics \& \\ Communication, NIIST, Bhopal
}

\begin{abstract}
This work gives an over view of IP telephony and explain the existing difficulties in implementing VoIP services \& migrate the TDM base telephony to IP telephony. When the architecture is deployed in IP based network. The causes of these problems are explained with three approaches used by carrier to solve interoperability. They are TDM base telephony to IP telephony by using soft switch and voice gateway, using different audio codec's for better voice quality, band width used in IP telephony.
\end{abstract}

\section{General Terms}

Routing, Rate, Currentcalls, Server

\section{Keywords}

SIP-Trunking, TDM, IP-PBX, Softswitch, softphone

\section{INTRODUCTION}

There is a technology used by Telecom Provider for migrate voice services from traditional TDM networks to new VoIP networks. There are many type of VoIP solution to provide voice services but this paper is working on SIP Trunking Technology. SIP Trunking: Unlike in traditional telephony, where bundles of physical wires were once delivered from the service provider to a business. A SIP Trunk allows a company to replace these traditional fixed Public Switched Telephone Network [PSTN] line with PSTN connectivity via a SIP Trunking service provide on the internet.

In figure, The PBX is located on the internal network. The PBX must have a SIP enabling trunking interface. It can either be an IP - based PBX communicating to all end points over IP or it may just as well be a traditional TDM PBX. The main requirement is that an interface for SIP Trunking connectivity is available over the internet. The ITSP [Internet Telephone Switch Network] providesconnectivity to the PSTN for communication with mobile and fixed phones.[1]

The IP telephone techniques is widely used in future because of its special features and cost reducing benefit to the company, In the traditional TDM base telephone is taking more space and provide fixed requirement line. If customer needed more telephone line then Service Provider revises his process to the start point to the end point but in the SIP Trunking there is no requirement to the new or more set up because of sip trunking data and voice both are transfer at the same line. Service provider just provide the fiber optics cable to the customer and according to the requirement of the customer SP's provide the channel and when customer need more channel's then SP's just increase the channel capacity and according to channel they have to maintain his bandwidth of the internet. In TDM base telephone lot's of bandwidth is waste if there is not more traffic at every time but in the IP Telephony by using SIP trunking that problem will be resolve and also save the bandwidth of the internet that also reduce your cost.In the past there are too many technology for using telephone service but in the VoIP network they reduce cost of the call's and set up so it's generate maximum revenue to the company (SP's).

IP telephone is mainly used in corporate industry, Call center, small and medium enterprises that is used SIP Trunking for increase his revenue and provide better service to the customer. There is an evolution of technology used by Telecom Providers tending to migrate voice services from the traditional TDM networks to new VoIP networks, especially involving SIP-Trunking solutions. The number of this type of migrations is expected to grow dramatically at $106 \%$ Compound Annual Growth Rate (CAGR) in the next few years.

The introduction of new VoIP solutions at Enterprise level is a great opportunity to redesign and standardize services. SIPTrunking makes possible to implement new services (like Presence, videoconference, virtual Fax) accessible as "advanced communications as a service" and that can change customer perception and improve productivity. Collaboration and travels cost reduction.

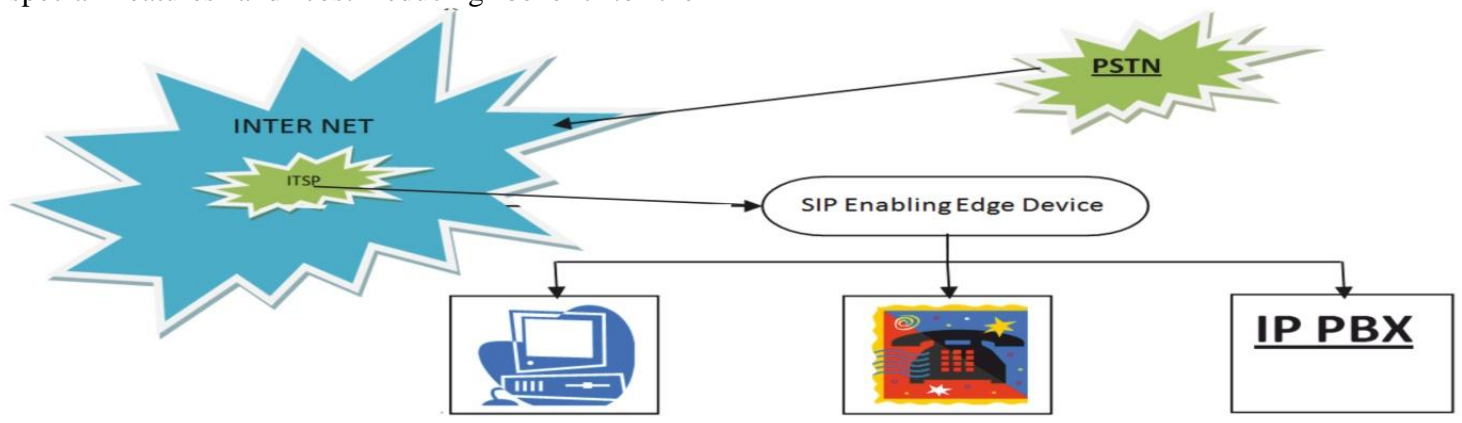

Fig. 1 : Typical SIP Trunking Solution [ 1] 


\section{LITERATURE SURVEY}

As per NenadKrajnovic from University of Belgrade in 2009[2] presenta article in Migration of TDM base telephone to the IP telephony network. In this article he's describe the migration of TDM network to IP base network. In telecom industry availability should be $99.999 \%$. For this availability there are many difficulties occurs in migrating TDM base telephony to IP base telephony, He's learn about three problem that is occur during this migration.

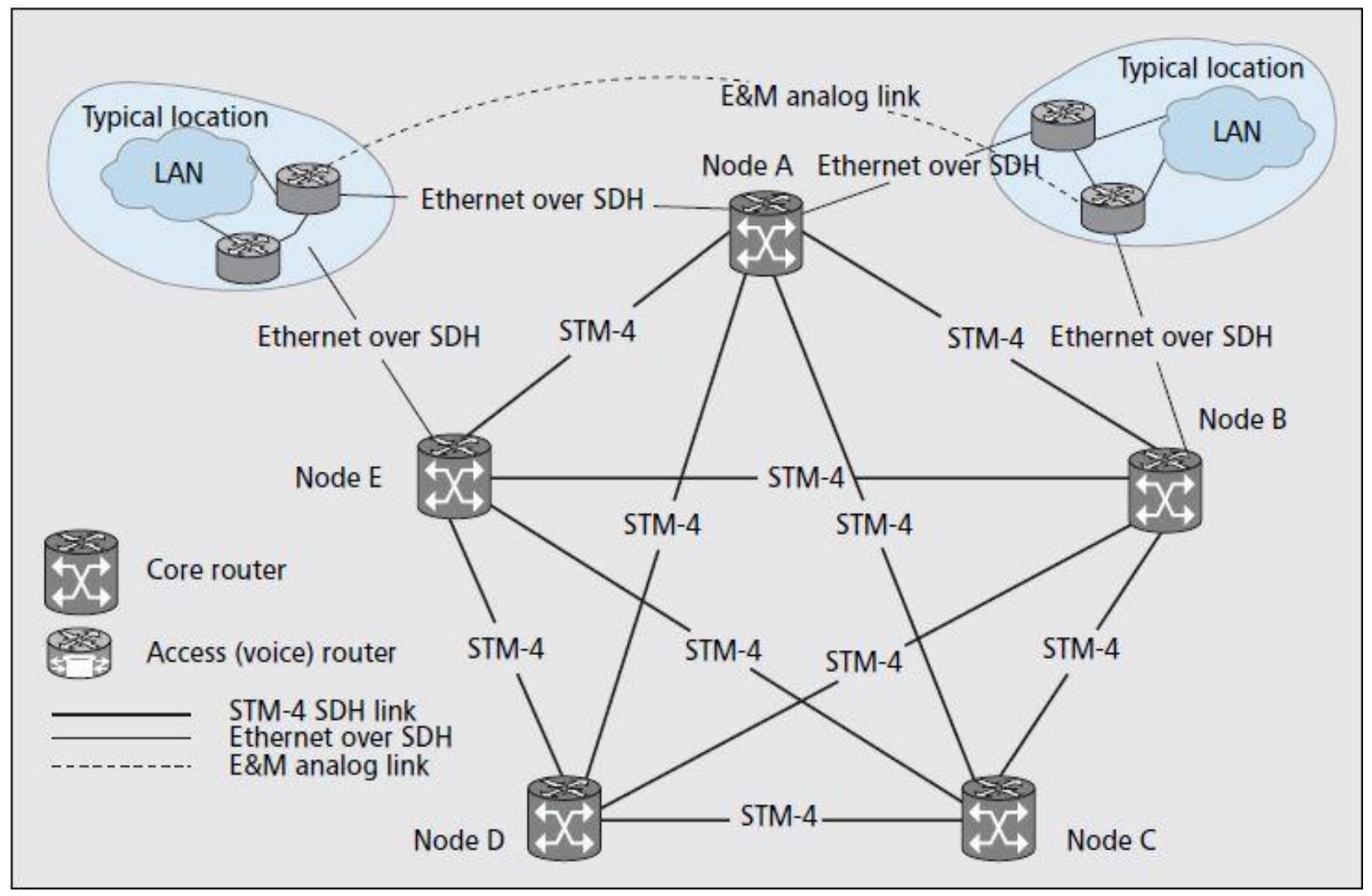

Fig 2: Logical architecture of the network[2]

1. Typical solution is to use SDH interface and channels. The problem with this approach, however, is the IP routers with $\mathrm{SDH}$ interfaces are relatively expensive. The SDH multiplexers are responsible for mapping the traffic to the appropriate virtual concatenated channels in the SDH multiplexers at the location of the routers. The generic framing procedure (GFP) and link capacity-adjustment scheme (LCAS) were used for flexible configuration and reconfiguration of the virtual concatenated SDH channels. Virtual local area network (VLAN) tagging was used for multiplexing of different links toward remote locations on the interface between SDH multiplexers and core routers. To achieve 99.999 percent availability. For this he is using two

switches which is used to provide 99.999 percent availability to the network if one switch link is down then second switch

will work as a first switch. All the traffic routing is mapped in access router and if both redundant link fail simultaneously then access router (which plays the role of a voice gateway and therefore, can be called a voice router) routes voice call traffic over the E\&M link connected to the neighboring site. Where it is converted to the IP destination end point (the end user): thus, end users do not experience an interruption in service. In such a design, at least three independent paths exist between any two phones in the network: Two paths over an IP network and a third path using legacy voice channels (the E\&M links).
2. Another problem emerged from the integration of the existing newer PBXs, particularly concerning the interconnect protocols. By NENAD KRAJNOVIC The two viable options for PBX interconnect was the Q-signaling (Q.Sig) protocol and Session InitiationProtocol (SIP) trunking. He's taking first option by the analysis of published information revealed that Q.Sig has the broadest set ofservices for which proven implementations exist. The great advantage in this case was that theclient mainly requested just the simpleestablishingof voice calls. In the end, it was decided to use E1 $(2 \mathrm{Mb} / \mathrm{s})$ links and the Q.Sig protocol as the interconnection between most of these PBXs. As per NENAD KRAJNOVIC despite published standards, there was no guarantee that voice telephony using VoIP/SIP interconnection would work between PBXs from different vendors. Investigation showed that successful interoperability depended on notonly the vendors and software versions of the PBX VoIP/SIP modules, but also on the specific telephony services and functions.

3. The next significant problem that was encountered was the design of a softswitch that could control the entire IP telephony network. Because of the requirement that the network have five nines (99.999) of availability, the client insisted on having two softswitches capable of operating in either active/active or active/standby mode. Additional softswitch requirements included the ability to support 15,000 heterogeneous end points (IP phones, lines terminated 
on voice routers, and lines terminated on hybrid PBXs), and the capacity to handle 60,000 busy hour calls completed. Network control was divided between two softswitches. If one of the softswitches failed, the remaining one would take control of the entire network without interrupting established calls. The maximum switchover time must not exceed $6 \mathrm{~s}$ for critical users. The softswitch is responsible for routingphone calls and defining the termination pointsfor calls. The IP routing layer, in combinationwith MPLS, is responsible for IP traffic routingin the network. The IP routing layer handles

responsible for call signaling and uses the survivable remotesite telephony (SRST) feature in the Cisco Internetworking Operating System (IOS).

As per ShIZhi-yong, ZHANG Zhen-yu, LI Hong-liang in 2009[1] is present a paper that is Design \& Simulation of Trunking Communication System in TD-SCDMA Network Based on SIP Protocol. In this paper he's describe a trunking communication system which is a branch of mobile communication, and is mainly applied in the special occation, such as dispatching. In this technology service provider can generate group call's, group multimedia message. It describe framework of the trunking communication system base on TD-SCDMA. For connecting a call in a trunking communication there are many difficulties occurs to generate failureof IP links and the switching of traffic toredundant IP links. In this hybrid solution, most calls use VoIP protocols and the IP network for transport. In the case where the end point of a call is not directly connected to the IP network, the softswitch is responsible for choosing the voice gateway for that call and for handling the in-band call signaling for the non-IP telephonenetwork. In the case of IP link failures, the softswitch predefines the paths through local voice routers and voice links (E\&M, E1)[3]. In those situations (i.e., no IP connectivity toward softswitch), the local voice router is

the group call or member's login. He's learn about two problem that is occurred during generating a call.

1.Trunking Subsystem: The trunking subsystem is the core part of realizing trunking communication services. It includes the TS, the trunking database, the group and list server, and the system dispatching console's is the total control point of the trunking call and fulfills the trunking dispatching handling which includes authenticating user, establishing trunking call (such as private call and group call), judging trunking PTT request. On the other hand, it receives trunking traffics from the reverse link and distributes them to the corresponding forward link according to the nature of the call character. TS connect with the system dispatching console by using Ethernet interface RJ-45.

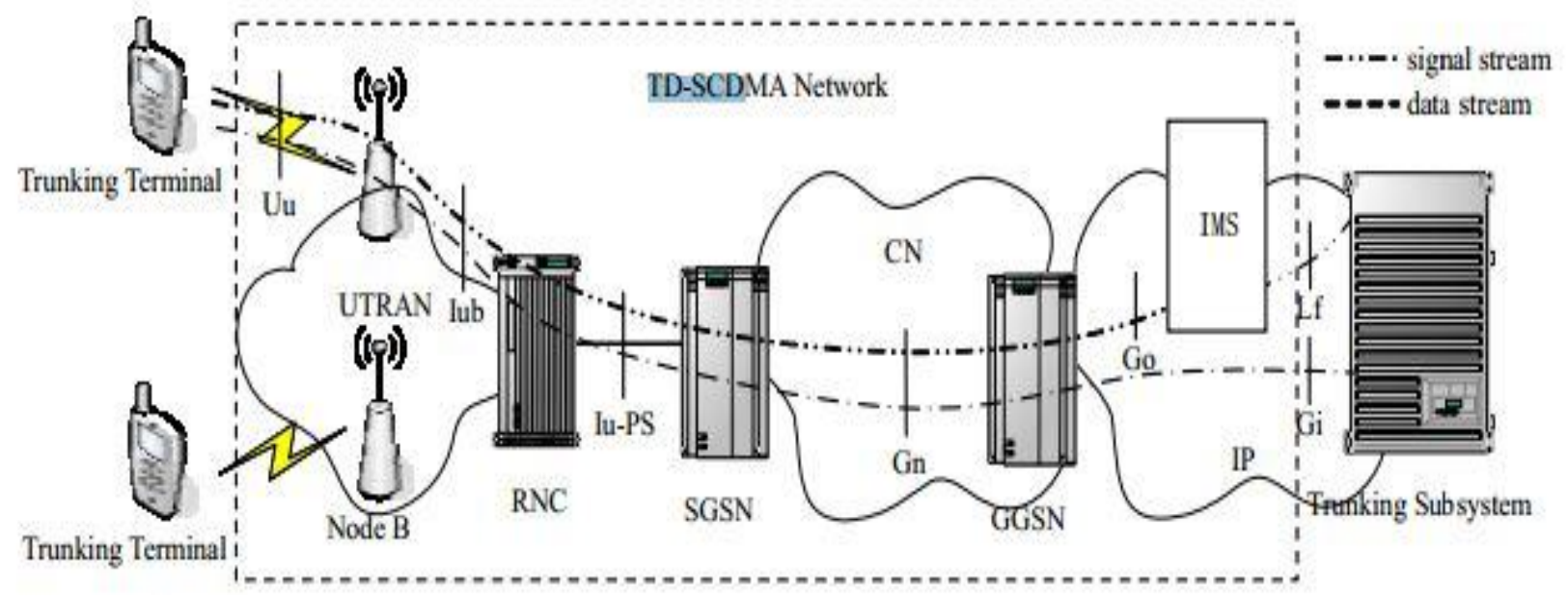

Fig 3: Trunking Communication System Framework[4]

The trunking database provides the group registration and the group member registration for user. It also can provide the local information of group member, the service authority record of group member, the statistics of completing call and the charging function. The group and list server is used to manage the group and the list, such as the address and the black-and white list.The system dispatching console adopts the wire console. It provides the control and the traffic management for trunking user. Its functions include the setting of the single call, the group call, the broadcasting call, the dynamic reorganization and the operation of forcing intervention or ending.

2. SIP APPLICATION: The delay time of connecting call would more than 6 seconds (about 6 to 8 seconds) if the connecting call mode of the public TD-SCDMA network in this digital trunking system. This paper do not consider these factors in here, such as the interruption and the power fluctuation of the system. The dispatching services will be seriously affected by the delay time of connecting call. The scheme of combining the mode of early establishing session and SIP signaling protocol is adopted for reducing the system
delay.SIP is a protocol based on text. It is used to control sessions of voice services and multimedia services in IP network. Its basic functions include the establishment, release and revises of communication session and the negotiation of TT's Communication ability. 3GPP clearly gave that SIP would be use as the control protocol of the core network after R5 version because it has the flexibility, the scalability and is still at the stage of development and improvement. SIP network adopts the $\mathrm{C} / \mathrm{S}$ (client/server) mode based on Internet. The client sends requests to server and establishes connection with server. The server processes the requests of the client and returns responses to the client. There are four basic servers in the communication of SIP, such as user agent server, proxy server, redirect server and registrar. The main purpose of using SIP is to establish session between two client proxies. The client proxies include the client sending SIP request and the server receiving the SIP request. SIP is always together with the session description protocol (SDP), and it is also said that SDP is used to describe the ongoing session. SIP information is transmitted by UDP or TCP. SIP is also a protocol based on processing affair. One SIP affair is made up by single request and all responses of this request. The session 
which is established by SIP includes request affairs and response affairs.[5][6]

As per Ivan Gaboli and Virgilio Puglia Kaleidoscope: Beyond the internet in 2010[7] is present a paper that is SIP TRUNKING THE ROUTE TO THE NEW VOIP SERVICES in this paper they describe the new technology which is most important in the future of telecommunication industry that is VoIP services. In this service most of the telecom provider is used a VoIP technology in the telecom industry, they suggest to the telecom provider that they use SIP trunking technology in his service which is very cost reducing technology and it is very suitable to the all customer's. They learn about market approach ad SIP signaling model and there they are found some difficulties to achieve prefect signaling.
1.SIP BUSINESS TRUNKING ARCHITECTURAL MODEL: The SIP Business-Trunking reference architecture is a five level hierarchical architecture divided into two domains: Public and Private.

-The Public Domain has two levels represented by:

- Class 5 softswitches (SSW-C5), that are the first level of the network model;

- Network Border-Elements (second level) that is the frontier of the public domain towards customers.

- The Private Domain has three hierarchical levels:

- Corporate Border-Element, the frontier towards the public network;

- $\quad$ Corporate Switching Node (CSN);

- IP-PBXs

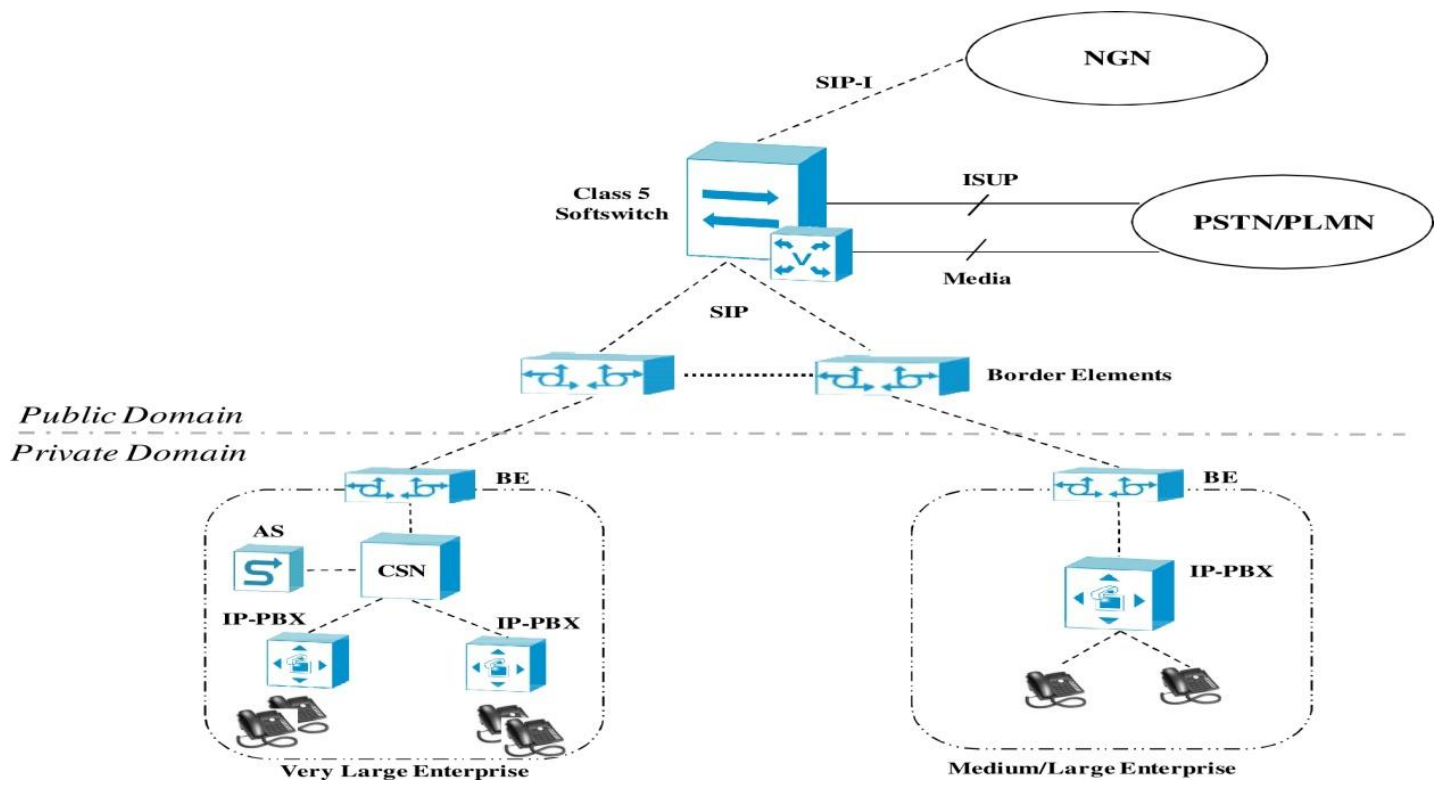

Fig 4: NETWORK Architectural Model[7]

The SSW-C5 are generally connected northbound to the SP's transit network (Class-4 Exchange) via ISUP or via SIP-I/SIP$\mathrm{T}$ whereas southbound SSW-C5 provides a SIP User to Network Interface (UNI) that allows the end user access to the PSTN/PLMN; the Business-Trunking UNI is defined combining a set of RFCs with appropriate policy defined by the carrier.

These devices provide termination and re origination of both signaling and media between Public and Private Domain. The $\mathrm{CSN}$ can be inserted in the architecture when the private VoIP network is very large and there are benefits having a local session routing capabilities.

1. MARKET APPROACH: Waiting for an efficient standardization of SIP, the SPs have two possibilities:

- Full Jacket (FJ) :If the volume (number of enterprise customers) is high, the SP can define some prepackaged solutions. This approach is usually utilized by the incumbent Carrier.

- Customer Tailored (CT): The SP tries to satisfy all the customer's requests without imposing technical limitations or prepackaged service bundles.
As per Pablo Montoro, Eduardo CasilariDpto.Tecnologia Electronica, University of Malaga (Spain) in 2009[8] is present a paper that is A Comparative Study of VoIP Standards with Asterisk is compare the performance of the Most extended protocols for VoIP are H.323, SIP and IAX. They compare this protocol working in Codec, Processor, Memory Utilization and Bandwidth consumption.There are four different type of codec that is high quality codec (g.711, A-law), an intermediate bandwidth codec (g.726), a lowbandwidth codec (gsm) and an ultra light codec (lpc10). As comparison of this coding chart as given below:

Table 1: PROPERTIES OF EMPLOYED CODEC [8]

\begin{tabular}{|l|l|l|l|}
\hline Codec & Bit rate & Frame size & Data size per frame \\
\hline g.711 & $64 \mathrm{kbps}$ & $20 \mathrm{~ms}$ & 160 bytes \\
\hline g.726 & $32 \mathrm{kbps}$ & $20 \mathrm{~ms}$ & 80 bytes \\
\hline gsm & $13 \mathrm{kbps}$ & $20 \mathrm{~ms}$ & 33 bytes \\
\hline lpc10 & $2.4 \mathrm{kbps}$ & $22.5 \mathrm{~ms}$ & 7 bytes \\
\hline
\end{tabular}


Processor: SIP and IAX work mostly as a same but in this protocol there is some difference for using processor utilization that chart as given below:

Table 2: Processor Utilization [8]

\begin{tabular}{|c|c|c|c|c|}
\hline Protocol & Codec & 10 calls & 20 calls & 30 calls \\
\hline SIP & g.711 & $2.36 \%$ & $4.64 \%$ & $7.12 \%$ \\
\hline SIP & g.726 & $2.13 \%$ & $4.46 \%$ & $6.99 \%$ \\
\hline SIP & gsm & $2.58 \%$ & $4.55 \%$ & $6.96 \%$ \\
\hline SIP & $\operatorname{lpc10}$ & $1.92 \%$ & $3.61 \%$ & $5.45 \%$ \\
\hline IAX & g.711 & $3.65 \%$ & $7.46 \%$ & $11.99 \%$ \\
\hline IAX & g.726 & $3.61 \%$ & $7.30 \%$ & $11.65 \%$ \\
\hline IAX & gsm & $3.62 \%$ & $7.36 \%$ & $11.73 \%$ \\
\hline IAX & $\operatorname{lpc10}$ & $2.90 \%$ & $5.77 \%$ & $9.12 \%$ \\
\hline IAX(Trunking) & g.711 & $2.11 \%$ & $4.02 \%$ & $5.56 \%$ \\
\hline IAX(Trunking) & g.726 & $2.06 \%$ & $3.90 \%$ & $6.46 \%$ \\
\hline IAX(Trunking) & gsm & $1.95 \%$ & $3.85 \%$ & $6.29 \%$ \\
\hline IAX(Trunking) & $\operatorname{lpc10}$ & $1.62 \%$ & $3.71 \%$ & $4.89 \%$ \\
\hline
\end{tabular}

Memory Utilization: In SIP and IAX memory utilization of both the protocol is range between $33 \mathrm{MB}$ to $42 \mathrm{MB}$. The comparison chart is given below:

Table 3: Memory Utilization [8]

\begin{tabular}{|c|c|c|c|c|}
\hline Protocol & Codec & 10 calls & 20 calls & 30 calls \\
\hline SIP & g.711 & 33.38 & 38.23 & 42.09 \\
\hline SIP & g.726 & 34.38 & 38.23 & 42.05 \\
\hline SIP & gsm & 34.04 & 37.96 & 41.81 \\
\hline SIP & $\operatorname{lpc10}$ & 34.42 & 38.25 & 42.06 \\
\hline IAX & g.711 & 33.93 & 37.06 & 40.44 \\
\hline IAX & g.726 & 33.70 & 36.89 & 40.29 \\
\hline IAX & gsm & 33.80 & 36.96 & 40.08 \\
\hline IAX & $\operatorname{lpc10}$ & 33.64 & 36.82 & 40.02 \\
\hline IAX(Trunking & g.711 & 33.82 & 36.84 & 40.01 \\
\hline IAX(Trunking & g.726 & 33.74 & 36.86 & 40.03 \\
\hline IAX(Trunking & gsm & 33.65 & 36.86 & 40.16 \\
\hline IAX(Trunking & lpc10 & 33.70 & 36.84 & 40.00 \\
\hline
\end{tabular}

OBTAINED BANDWIDTH: IAX protocol obtained low bandwidth as compare to the SIP, Comparison Chart is given below:

Table 4: Obtained Bandwidth [8]

\begin{tabular}{|l|l|l|l|l|}
\hline Protocol & Codec & 10 calls & 20 calls & 30 calls \\
\hline SIP/RTP & g.711 & $\mathbf{8 6 . 0 5}$ & $\mathbf{8 6 . 0 0}$ & $\mathbf{8 6 . 0 0}$ \\
\hline SIP/RTP & g.726 & $\mathbf{5 3 . 9 5}$ & $\mathbf{5 4 . 0 0}$ & $\mathbf{5 4 . 0 0}$ \\
\hline SIP/RTP & gsm & $\mathbf{3 5 . 0 4}$ & $\mathbf{3 5 . 0 7}$ & 35.00 \\
\hline SIP/RTP & lpc10 & 19.50 & 19.52 & 19.51 \\
\hline IAX & g.711 & $\mathbf{8 2 . 9 6}$ & $\mathbf{8 3 . 0 0}$ & $\mathbf{8 3 . 0 0}$ \\
\hline IAX & g.726 & $\mathbf{5 0 . 8 2}$ & $\mathbf{5 1 . 0 0}$ & $\mathbf{5 0 . 6 7}$ \\
\hline
\end{tabular}

\begin{tabular}{|l|l|l|l|l|}
\hline IAX & gsm & 31.93 & 31.95 & 31.93 \\
\hline IAX & lpc10 & 19.26 & 19.26 & 19.26 \\
\hline IAX(Trunking) & g.711 & 70.21 & 69.25 & 69.00 \\
\hline IAX(Trunking) & g.726 & 36.75 & 36.49 & 35.67 \\
\hline IAX(Trunking) & gsm & 17.88 & 16.87 & 16.54 \\
\hline IAX(Trunking) & lpc10 & 6.35 & 5.35 & 5.12 \\
\hline
\end{tabular}

\section{CONCLUSION AND FUTURE WORK}

The migration towards IP telephony process is very difficult and required careful analysis of every step and the close cooperation of equipment and solution venders. Applying general principles and depending on compatibility and interoperability through standards when designing an IP telephony network is not sufficient to guarantee a successful implementation: extensive interoperability testing is an absolute requirement. Future scope is to eliminate the difficulties of migration towards IP telephony by the use of SIP Trunking Technique and reduce cost of the total project for the Service Provider.

\section{REFERENCES}

[1] Janne Magnusson, "SIP Trunking Benefits and Best Practices" White Paper.

[2] NenadKrajnovic "The Design of a Highly Available Enterprise IP Telephony Network for the Power Utility of Serbia Company," IEEE Commun. Mag. Vol. 47, issue. 4, Apr. 2009, pp. $118-122$.

[3] A. D. Keromytis, "A comprehensive survey of Voice over IP security research", IEEE Communications Surveys \& Tutorials, vol. 14, no. 2, 2012, Page(s): 514537.

[4] Ivan Gaboli, Virgilio Puglia, "SIP TrunkingThe Route to the New VoIP Services," Kaleidoscope: Beyond The Internet? - Innovation for Future Network and Services, ITU-T, 2010, pp. $1-8$.

[5] Tuong Le, S. Cook, G. Kuthethoor, P. Sesha, G. Hadynski, D. Kiwior, and D. Parker. "Performance analysis for SIP based VoIP services over airborne tactical networks, " In Aerospace Conference, 2010 IEEE, Page(s): 1-8.

[6] Mohamed Boumezzough, N. Idboufker, and A Ouahman. "Evaluation of SIP Call Setup Delay for VoIP in IMS, " In Advanced Infocomm Technology, Springer Berlin Heidelberg, 2013, Page(s): 16-24.

[7] Shi Zhi-yong ; Zhang Zhen-yu ; Li Hong-liang ;Zhang Zhen-yu "Design and Simulation of Trunking Communication System in TD-SCDMA Network Based on SIP Protocol" Information Engineering and Electronic Commerce, 2009. IEEC'09. International Symposium on Digital Object Identifier: 10.1109/IEEC.2009.107 Publication Year: 2009 , Page(s): 481 - 485.

[8] Montoro, P. ;Casilari, E. "A Comparative Study of VoIP Standards with Asterisk" Digital Telecommunications, 2009. ICDT '09. Fourth International Conference on Digital Object Identifier: 10.1109/ICDT.2009.8 Publication Year: 2009 , Page(s): $1-6$. 